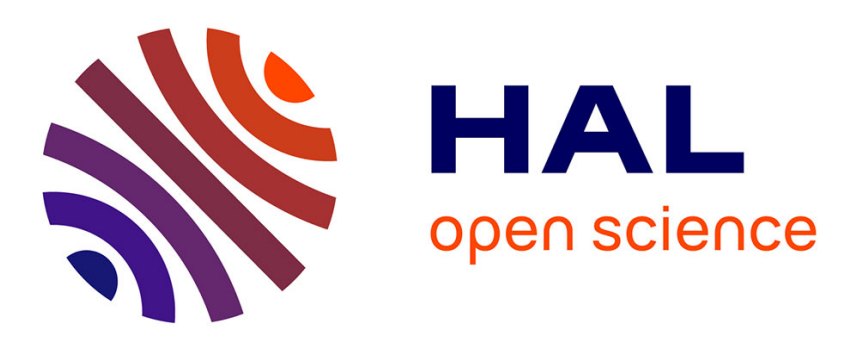

\title{
Ask not what's Inside your head, but what your head is inside of (Mace, 1977)
}

Benoît G. Bardy, Bruno Mantel

\section{To cite this version:}

Benoît G. Bardy, Bruno Mantel. Ask not what's Inside your head, but what your head is inside of (Mace, 1977). Intellectica - La revue de l'Association pour la Recherche sur les sciences de la Cognition (ARCo), 2006, 10.3406/intel.2006.1328 . hal-02939708

\section{HAL Id: hal-02939708 https://hal.science/hal-02939708}

Submitted on 15 Sep 2020

HAL is a multi-disciplinary open access archive for the deposit and dissemination of scientific research documents, whether they are published or not. The documents may come from teaching and research institutions in France or abroad, or from public or private research centers.
L'archive ouverte pluridisciplinaire HAL, est destinée au dépôt et à la diffusion de documents scientifiques de niveau recherche, publiés ou non, émanant des établissements d'enseignement et de recherche français ou étrangers, des laboratoires publics ou privés. 


\title{
Ask not what's inside your head, but what your head is inside of (Mace, 1977)
}

\author{
Benoît G. BARDY et Bruno MANTEL*
}

RESUME. Ce commentaire vise à éclairer les similitudes et différences existant entre le courant Noéien de l'énaction défendu par Lenay et critiqué par Jacob, et l'approche écologique de la perception établie par Gibson et développée depuis un demi-siècle. Les points abordés concernent la notion de représentation, la distinction entre sensation et information, le couplage sensori-moteur, et l'inadéquation de la notion de copie d'efférence.

Mots clés: Approche écologique - Copie d'efférence - Couplage - Enaction Information - Sensation

\begin{abstract}
This commentary highlights the similarities and differences between on the one hand the (Noeian-viewed) concept of enaction defended by Lenay and criticized by Jacob, and on the other hand the Gibsonian, ecological approach to perception and action. In this context, we address the notions of representation, sensation and information, sensori-motor coupling, and inadequacy of efferent copy.
\end{abstract}

Key words: Ecological approach - Coupling - Efferent copy - Enaction - Information Sensation

Sur la question de l'externalisme et de l'énaction en philosophie de la perception, les articles-cible de Lenay (2006) et Jacob (2006) peuvent pour le lecteur apparaître complémentaires. A partir d'une lecture Noëienne de l'énaction (Noë, 2004 ; O’Regan \& Noë, 2001) et en s'appuyant sur ses propres travaux concernant la localisation spatiale, Lenay promeut une conception sensorimotrice de la perception soulignant le rôle de l'action dans la création des sensations et la construction de l'expérience perceptive. Jacob critique cette lecture de l'énaction, en particulier sa difficile réconciliation avec quelques expériences perceptives bien documentées comme la présence perceptive, la permanence des couleurs, ou l'anticipation des conséquences sensorielles de l'action. Le format du commentaire prohibe une analyse détaillée des points évoqués par les deux auteurs, aussi nous limiterons nous à souligner trois aspects, forcément caricaturaux, concernant les mécanismes de couplage entre la perception et l'action.

\section{SENSATION, INFORMATION, ET COUPLAGE PERCEPTION-ACTION}

Tout d'abord, nous saluons l'effort fait par Lenay pour s'écarter de la position internaliste et computationaliste en sciences cognitives visant à attribuer la cohérence de nos percepts et l'adaptabilité de nos mouvements à la cohérence et à l'adaptabilité de mécanismes sui generi - les représentations — qui constituent une hypothèque sur l'intelligence (Kugler et al., 1980). Considérer a priori les représentations, perceptives ou motrices, comme des mécanismes

\footnotetext{
* Benoît Bardy, Université Montpellier-I, Efficience \& Déficience Motrices (UFR STAPS), 700 Avenue du Pic Saint Loup, 34090 Montpellier, France, Tel: +33 (0)467 415 767, Fax: +33 (0)467415 704, benoit.bardy@univ-montp1.fr, bruno.mantel@univ-montp1.fr, http://www.edm.univ-montp1.fr. Support : Réseau européen Enactive Interfaces (IST \#002114), http://www.enactivenetwork.org/
} 
fondamentaux propres à l'espèce humaine minimise en effet le rôle des lois physiques et biologiques en vertu desquelles une perception de la réalité est accessible. Le point de départ de la théorie Gibsonienne, que semble partager Lenay consiste à l'inverse à prendre au sérieux le caractère insécable de la relation entre l'observateur et son environnement, et la réciprocité qui existe entre Percevoir et Agir. La phrase bien connue de Gibson « Nous devons percevoir pour bouger, mais nous devons aussi bouger pour percevoir » (1979, p. 223, trad.) traduit simplement cette mutuelle dépendance entre les forces internes produites par l'observateur et les flux informationnels, à la fois conséquences et causes du mouvement. Conséquences car le déplacement de l'observateur produit une transformation unique de la configuration optique (Gibson, Olum \& Rosenblatt, 1955), que l'on peut exprimer par la relation : Flux $=\underline{\mathrm{f}}$ (Force) (équation 1). Causes car les forces à produire sont souvent calibrées, en direction ou intensité, en fonction de ce flux optique [Force $=\mathrm{g}$ (Flux)]. La figure 1 rappelle ce cycle perception-action (Kugler \& Turvey, 1987) au cours duquel les deux composantes se déterminent mutuellement, et doivent être considérées comme deux modes complémentaires de couplage : un couplage à basse énergie entre l'action et la perception - les masses mises en jeu dans les flux cinématiques sont négligeables - et un couplage à haute énergie entre la perception et l'action — les masses mises en jeu dans les flux cinétiques sont importantes.

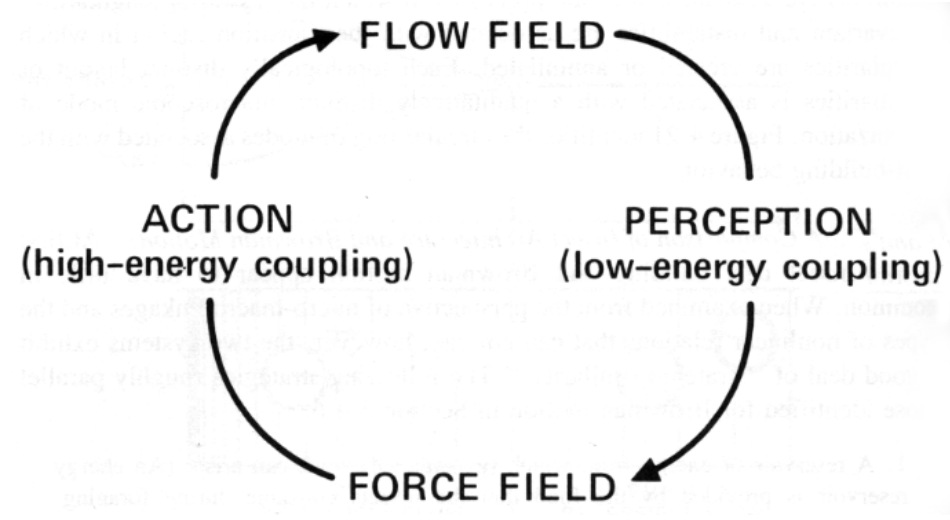

Figure 1. Le cycle perception-action (figure originale de Kugler \& Turvey, 1987).

Un aspect essentiel de ce couplage est que la perception prend sa source dans la structure spatio-temporelle des configurations ambiantes (optique, inertielle, acoustique, etc...) qui viennent stimuler nos récepteurs, non dans la stimulation de nos récepteurs (sensations). Pour Gibson, les persistances et les changements contenus dans les structures ambiantes et induits par l'état de l'observateur sont spécifiques de cet état et constituent de ce fait des informations potentielles pour la perception. La spécification est ici le processus qui lie de manière univoque les patrons ambiants d'énergie et la réalité physique que ces patrons révèlent. La manière dont la réalité est spécifiée dans les flux énergétiques qui viennent stimuler nos récepteurs ne relève pas à proprement parler de la biologie ou de la psychologie. Ces flux dépendent des lois physiques de propagation, d'absorption, ou de réflexion de l'énergie, non des caractéristiques de nos récepteurs sensoriels ou de nos mécanismes cognitifs. En ce sens, 
la théorie écologique du couplage entre l'action et la perception, dont les bases remontent aux années 1950, s’affranchit du problème de la représentation des objets et événements. Si les propriétés de l'interaction entre l'observateur et l'environnement sont spécifiées dans la structure des énergies ambiantes, une thèse que la physique ne peut réfuter, alors ces propriétés n'ont pas à être représentées, si ce n'est physiquement dans l'interaction. Elles constituent dès lors des informations potentielles pour la perception (voir Bardy, 2003, pour les détails). L'externalisme fort défendu par Lenay semble ainsi largement inspiré de l'approche écologique.

\section{LOIS DE CONTROLE DU MOUVEMENT VS. LOIS DE CONTINGENCES SENSORI-MOTRICES}

Ce principe de causalité circulaire, s'il est pris au sérieux, conduit le chercheur à identifier les lois par lesquelles les variables perceptives générées participent à la régulation de l'action. La façon dont ces variables perceptives modulent les variables motrices de l'action en cours est la problématique générale des lois de contrôle (Warren, 1988). Dans un article immensément cité ayant jeté les fondations de l'approche non représentationnelle de la perception, Gibson (1958) proposa cinq formules décrivant le contrôle de la locomotion, gouvernant les actions d'initiation, d'arrêt, d'atteinte du but, de freinage, d'évitement d'obstacle, etc... Il nomma un peu plus tard ces formules règles, précisant que ces dernières " ne sont pas des commandes en provenance du cerveau mais émergent du système animal - environnement » (Gibson, 1979, p. 232). Warren (1988) les nomma ensuite lois pour souligner leur existence irréductible et systématique dans la relation entre l'information et le comportement. Les lois de contrôle sont des relations entre les variables informationnelles (i.e., optiques, inertielles, acoustiques, etc..) et les paramètres libres du système d'action. Elles peuvent être exprimées par la relation Force $_{\text {ext }}+$ Force $_{\text {int }}=$ g (Flux) (équation 2), caractéristique de l'interaction animal - environnement, où Force ${ }_{\text {int }}$, et Force $e_{\text {ext }}$ sont spécifiées dans les flux énergétiques.

Le concept de contingences sensori-motrices créé par Noë et repris par Lenay et Jacob semble très proche des notions de lois de contrôle proposées par Gibson (1958) et Warren (1988). ${ }^{1}$ Si tel n'est pas le cas, les différences entre ces deux notions nous semblent devoir être précisées par les auteurs.

\section{ANTICIPATION DES CONSEQUENCES SENSORIELLES ET COPIE D'EFFERENCE}

L'équation 2 est à la base de la théorie de la perception visuelle de Gibson. Néanmoins, elle est insuffisante pour caractériser le contrôle de l'action car les forces qui la composent et (qui spécifient le flux optique) sont les forces totales qui agissent sur le corps, c'est-à-dire les forces internes - celles qui sont appliquées par l'observateur - ajoutées aux forces externes - celles qui lui sont imposées, comme le vent ou la gravité par exemple. Comment dans ce cas l'observateur désambiguïse-t-il dans le flux les forces appliquées - celles qu'il doit réguler - des forces imposées ? La réponse classique à cette question, comme le souligne Jacob, consiste à suggérer l'existence d'une copie de

\footnotetext{
1 Une littérature expérimentale abondante existe depuis plus de deux décennies, visant à tester l'existence et le rôle fonctionnel de ces lois de contrôle dans de très nombreuses situations sensorimotrices (marcher, courir, sauter, voler, conduire, saisir, enjamber, etc...).
} 
la commande motrice, où copie d'efférence (Von Holst, 1954), préparant le système nerveux aux conséquences sensorielles du mouvement. La suggestion est séduisante car cette copie des ordres moteurs offre à l'observateur la possibilité d'anticiper les conséquences optiques de son déplacement, à partir des commandes motrices connues. Pour des systèmes sensori-moteurs à faible nombre de degrés de liberté (comme l'œil par exemple), la copie d'efférence joue peut être un rôle important dans l'anticipation des conséquences du mouvement. Pour des systèmes sensori-moteurs plus complexes, tel que le système postural ou locomoteur, ou encore le système de capture ou de saisie manuelle, les chances que cette copie de la commande joue effectivement ce rôle restent inconnues, et ce pour une raison très simple mise en évidence par Bernstein (1967). Il n’existe en général pas de lien univoque entre la commande motrice et le mouvement. Une même commande peut produire des mouvements différents, et des commandes différentes peuvent produire un mouvement identique. De la même façon, à une même force peut correspondre des mouvements différents, et à des forces différentes peuvent correspondre le même mouvement. Les causes de cette non-univocité sont nombreuses et concernent par exemple l'anatomie (les propriétés visco-élastiques des muscles considérés, la position initiale des segments), les influences inertielles mutuelles de ces segments, la fatigue musculaire, l'entraînement, ou encore la fluctuation des forces externes en vigueur. Même chez la mouche, un peu de poussière sur une aile peut altérer considérablement la relation entre l'activité musculaire et le mouvement résultant. Cette équivocité est d'autant plus importante que les mouvements sont complexes et mettent en jeu un nombre important de degrés de liberté. La copie d'efférence s'avère ici insuffisante pour anticiper les conséquences sensorielles du mouvement. La proposition de Gibson pour éviter cet écueil consiste à mettre l'accent une fois de plus sur la réciprocité entre percevoir et agir: puisque la structure optique spécifie la relation courante entre l'organisme et l'environnement, alors un changement dans cette relation, qu'elle qu'en soit l'origine, intentionnelle ou extérieure, est spécifié par un changement dans le flux optique. Ce dernier peut ainsi être compensé par une modulation des forces internes de l'organisme (loi de contrôle). Ainsi, la production et la régulation du mouvement ne sont pas ici assurés par un mécanisme de réduction d'écart entre la commande motrice et l'anticipation des conséquences sensorielles du mouvement, mais par une modulation des forces internes sur la base d'une intention motrice optiquement spécifiée : $\Delta$ Force $_{\text {int }}=$ g ( $\Delta$ Flux) (équation 3, tirée de Warren, 1988). En ce sens, l'approche initiée par Gibson s'affranchit non seulement de la représentation du monde, mais aussi de celle des mouvements à produire et de leurs conséquences sensorielles. Si les variables de contrôle sont représentées, elles le sont ici physiquement, dans la relation entre l'observateur et l'environnement, et non symboliquement dans le système nerveux central.

\section{INVARIANCE, CHANGEMENT, INTERMODALITE}

L'emphase mise par Lenay (critiquée par Jacob) sur la notion d'invariant est là encore difficile à écarter des travaux de Gibson et de ses successeurs (voir Cutting, 1983, pour une revue complète). Empruntant aux mathématiques la notion de ce qui est laissé inchangé par une transformation, les structures énergétiques stimulant nos récepteurs sont qualifiées d'invariants (Michaels \& Carello, 1981). Ces invariants peuvent être structurels (e.g., constance de la forme d'un objet par exemple malgré le changement de point de vue) ou transformationnels (e.g., trajectoire de cet objet, caractéristique du mouvement 
qui l'anime). Les expériences princeps de Johansson (e.g., 1973) en sont une parfaite illustration : le mouvement de quelques marqueurs placés sur les articulations du corps humain contient de puissants indices sur la morphologie, le sexe, l’âge, voire l'intention de l'observateur.

Cette acceptation de l'importance des invariants dans la perception contraste (chez Lenay) avec la manière dont les invariants inter-sensoriels sont traités. Dans les expériences présentées, la perception semble monosensorielle, comme si les autres sens étaient éteints : «Il n’y a qu’un champ récepteur, donc seulement une sensation à chaque instant et donc là encore aucune spatialité intrinsèque du signal d'entrée » (p. 6). Cette assertion est certainement plausible si l'on ne considère que la seule information tactile au bout du doigt, mais peu réaliste si l'on accepte que TOUT mouvement est fondamentalement multimodal. Ainsi l'énergie inertielle, au travers des forces qui s'exercent (i.e., qui s'opposent au mouvement ou l'entraînent), lors des accélérations linéaires et angulaires, renseigne sur la position du bras. L'information sur la distance et/ou l'orientation de la cible est alors spécifiée par un invariant intermodal entre les dimensions tactiles et inertielles du paysage sensoriel.

Lorsque Lenay envisage l'existence de dimensions autres que tactiles au sein de l'expérience sensorielle, il ne les considère pas comme simultanées, donnant l'impression que les flux modaux sont au mieux indépendants et au pire séquentiels. Dans cette interprétation l'auteur semble oublier deux points fondamentaux. Premièrement, la perception est continuellement multimodale et la co-existence des informations proprioceptives et tactiles n'est pas fortuite, car elle découle directement de la relation entre l’individu et le monde réel. En outre, un même objet ou un même mouvement (du monde réel) participe simultanément à la structure de différentes énergies ambiantes : il existe donc des structures inter-énergétiques, c'est-à-dire des invariants qui s'étendent sur plusieurs énergies. Conséquemment, et c'est le deuxième point, cette covariation non-fortuite est informative (car spécifique du système individu/environnement, Stoffregen \& Bardy, 2001). Par exemple, nous avons récemment formalisé un invariant intermodal spécifiant la distance égocentrique entre les dimensions optiques et inertielles du paysage sensoriel (Mantel, Bardy \& Stoffregen, 2005). Nos résultats montrent que cette relation invariante est bien perçue et utilisée par l'être humain pour détecter la distance de préhension.

\section{REFERENCES}

Bardy, B.G. (2003). Le contrôle visuel des déplacements, in Delorme A. \& Flückiger M. (Eds.), Perception et réalité, Paris, DeBoeck.

Bernstein, N. (1967). The Co-ordination and Regulation of Movements, Pergamon Press.

Cutting, J.E. (1983). Four Assumptions about Invariance in Perception, Journal of Experimental Psychology: Human Perception and Performance, 9, pp. 310-317.

Gibson, J. J. (1958). Visually Controlled Locomotion and Visual Orientation in Animals, British Journal of Psychology, 49, pp. 182-194.

Gibson, J. J. (1979). The Ecological Approach to Visual Perception, Houghton-Mifflin.

Gibson, J. J., Olum, P. \& Rosenblatt, F. (1955). Parallax and Perspective during Aircraft Landings, American Journal of Psychology, 68, pp. 372-385.

Jacob, P. (2006). Portée et limite des théories énactives de l'expérience visuelle, Intellectica (ce numéro).

Johansson, G. (1973). Visual Perception of Biological Motion and a Model for its Analysis, Perception \& Psychophysics, 14, pp. 201-211. 
Kugler, P. N., Kelso, J. A. S. \& Turvey, M. T. (1980). On the Concept of Coordinative Structures as Dissipative Structures: I. Theoretical Lines of Convergence, in Stelmach G. E. \& Requin J. (Eds.), Tutorials in Motor Behavior, New York, John Holland, pp. 3-47.

Kugler, P. N., \& Turvey, M. T. (1987). Information, Natural Law, and the Self-assembly of Rhythmic Movement, Mahwah, NJ, Erlbaum.

Lenay, C. (2006). Enaction, externalisme et suppléance perceptive, Intellectica (ce numéro).

Mace, W. M. (1977). James J. Gibson’s Strategy for Perceiving: Ask not what's inside your Head, but what your Head is inside of, in Shaw R. E. \& Bransford J. (Eds.), Perceiving, Acting, and Knowing: Toward an Ecological Psychology, Mahwah, NJ, Erlbaum.

Mantel, B., Bardy, B. G. \& Stoffregen, T. A. (2005). Intermodal Specification of Egocentric Distance in a Target Reaching Task, in Heft H. \& Marsh K. L. (Eds.), Studies in Perception and Action VIII, Mahwah, NJ, Erlbaum, pp. 173-176.

Michaels, C. F. \& Carello, C. (1981). Direct Perception, Prentice-Hall.

Noë, A. (2004). Action in Perception, Cambridge, Mass., MIT Press.

O’Regan, J.K. \& Noë, A. (2001). A Sensorimotor Account of Vision and Visual Consciousness, Behavioral and Brain Sciences, 24, pp. 939-1031.

Stoffregen, T.A. \& Bardy, B.G. (2001). On Specification and the Senses, Behavioral and Brain Sciences, 24, pp. 195-261.

Von Holst, E. (1954). Relations between the Central Nervous System and the Peripheral Organs, British Journal of Animal Behavior, 2, pp. 89-94.

Warren, W. H. (1988). Action Modes and Laws of Control for the Visual Guidance of Action, in Meijer O. G. \& Roth K. (Eds.), Complex movement behaviour: 'The' motor-action controversy, Amsterdam, NL, North Holland, pp. 339-380. 